\title{
CARS and Raman microscopy of Alzheimer's Brain Tissue
}

\author{
Annika Enejder ${ }^{\mathrm{a}}$, Fredrik Svedberg ${ }^{\mathrm{a}}$, Lena Nyberg ${ }^{\mathrm{a}}$, and Jia-Yi Li ${ }^{\mathrm{b}}$ \\ ${ }^{a}$ Department of Chemical and Biological Engineering, \\ Chalmers University of Technology, Kemivägen 10, S-412 96 Göteborg, Sweden \\ ${ }^{b}$ Wallenberg Neuroscience Center, Department of Experimental Medical Science, \\ Lund University, S-221 84 Lund, Sweden
}

\section{INTRODUCTION}

Alzheimer's disease (AD) is a progressive neurodegenerative disorder without cure, characterized by the presence of extracellular plaques surrounded by dystrophic neuritis ${ }^{1}$. Fundamental understanding of the molecular processes involved is urgently needed in order to develop strategies to inhibit steps in the AD pathogenesis. Current understanding is that the senile plaques are composed of peptide fragments (Amyloid$\beta$ peptides) formed after the cleavage of the transmembrane protein amyloid protein precursor (APP) ${ }^{1}$. This knowledge relies primarily on biochemical analysis of the plaques in cell extracts together with fluorescence microscopy of tissue samples exposed to harsh preparation procedures and labeling. Thus, it is disputed to which extent the observations reported reflect the true biochemistry and morphology of AD brain tissue. By the powerful combination of Raman microspectroscopy, Coherent Anti-Stokes Raman Scattering (CARS) and 2-photon fluorescence microscopy, we show that the molecular composition of $\mathrm{AD}$ plaques is more complex and consequently the mechanisms behind their formation.

\section{MATERIAL \& METHODS}

Samples from human AD brain tissue (severe and end stages) originating from different brain banks (Lund and New York) were examined. From each sample, two subsets were formed; unstained slices for CARS and Raman microscopy as well as Thioflavin-S stained slices for 2-photon fluorescence (2PF) microscopy of the Amyloid- $\beta$ peptides. The slices, $\sim 40 \mu \mathrm{m}$ thick, were mounted on a cover slip in a small cell containing PBS solution. Three sets of data were collected: (i) simultaneously collected CARS and 2PF microscopy images of stained AD brain tissue samples, (ii) volume CARS microscopy images $\left(2845 \mathrm{~cm}^{-1}\right)$ and (iii) Raman microspectroscopy grids of fresh samples. The CARS and 2PF microscopy was conducted using a ps-pulsed laser system consisting of a Nd:VAN laser (picoTRAIN, High-Q Laser $\mathrm{GmbH}$ ) pumping an OPO (Emerald, APE $\mathrm{GmbH}$ ) and an inverted microscope (Nikon TE-2000) with a fast mirror scanning unit (Nikon C1) and a dual 
detection channel ${ }^{2}$. The Raman microspectroscopy was conducted using a continuous laser source at $532 \mathrm{~nm}$ coupled into an inVia Raman microscope (Renishaw Inc.). The combination of CARS and 2PF microscopy provided us with volume images of the 3dimensional distributions of lipids and Amyloid- $\beta$ peptides in the AD plaque region, which could be further confirmed at higher level of detail on fresh samples in the Raman microspectroscopy grids. Thus, the three techniques contributed with different and complementary aspects of the biochemistry and morphology of AD brain tissue not only limited to the Amyloid- $\beta$ aggregation process, thus, providing a more complete picture of the complex cascade of mechanisms behind the disease.

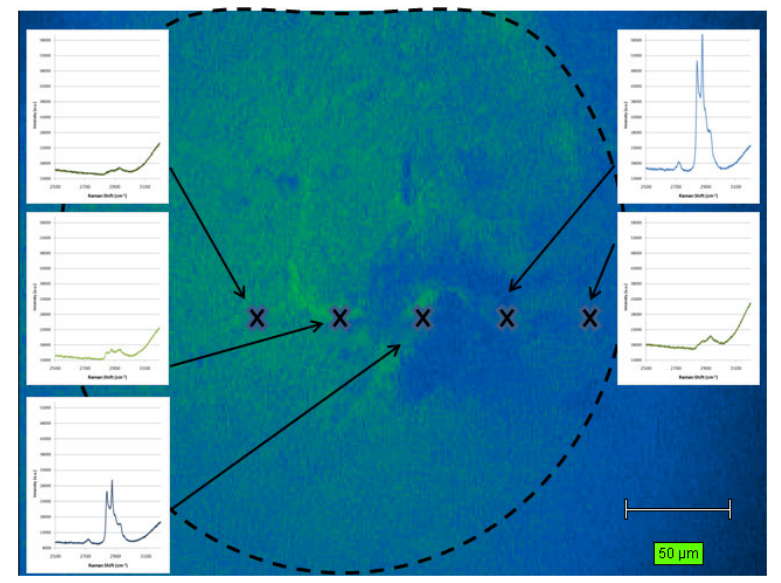

FIGURE 1. Transmission white light image of a region with AD plaques, highlighted by a dashed line. The Raman spectra shown (right and left) were collected at locations indicated by cross marks. Clear spectral differences characteristic of proteins and lipids can be observed, confirming that the molecular composition of AD plaques is more complex than current understanding. This is further confirmed by overlay CARS/2PF images of lipids and Amyloid- $\beta$ aggregates (data not shown).

\section{RESULTS \& CONCLUSION}

The simultaneously collected CARS and 2PF microscopy images (data not shown) indicate that $\mathrm{AD}$ plaques are associated with a complex distribution of Amyloid- $\beta$ aggregates and lipids. This is further confirmed by Raman spectra collected from the same sample using Raman microspectroscopy, as illustrated in Figure 1. This indicates that the well-known formation of Amyloid- $\beta$ aggregates is merely one of the processes involved in a complex cascade of molecular events underlying AD.

\section{ACKNOWLEDGMENTS}

The Raman microspectroscopy measurements were conducted in collaboration with K-analys, Uppsala, Sweden. This work was financially supported by the Swedish Research Council and the Swedish Dementia Foundation.

\section{REFERENCES}

1. R. B. Knowles, C. Wyart, S. V. Buldyrev, L. Cruz, B. Urbanc, M. E. Hasselmo, H. E. Stanley and B. T. Hyman, PNAS 96, 5274-5279 (1999)

2. A. Enejder, C. Brackmann, and F. Svedberg, IEEE J. Quantum Electron., Accepted (2010) 